\title{
The local structure fingerprint of dual orbital degeneracy lifting in a strongly correlated electron system
}

\author{
R. J. Koch ${ }^{1}$, R. Sinclair ${ }^{2}$, M. T. McDonnell ${ }^{3}$, R. Yu ${ }^{1}$, M. Abeykoon ${ }^{4}$, M. G. Tucker ${ }^{3}$, A. M. Tsvelik ${ }^{1}$, S. J. L. Billinge ${ }^{1,5}$, \\ H. D. Zhou' ${ }^{2}$, W.-G. Yin ${ }^{1}$, E. S. Bozin ${ }^{1}$
}

${ }^{1}$ Condensed Matter Physics and Materials Science Division, Brookhaven National Laboratory, Upton, NY 11973, US, ${ }^{2}$ Department of Physics and Astronomy, University of Tennessee, Knoxville, TN 37996, USA, ${ }^{3}$ Neutron Scattering Division, Oak Ridge National Laboratory, Oak Ridge, TN 37831, USA, ${ }^{4}$ Photon Sciences Division, Brookhaven National Laboratory, Upton, NY 11973, USA,

${ }^{5}$ Department of Applied Physics and Applied Mathematics, Columbia University, New York, NY 10027, USA

rkoch@bnl.gov

The local structure of $\mathrm{NaTiSi}_{2} \mathrm{O}_{6}$ is examined across its Ti-dimerization orbital-assisted Peierls transition at $210 \mathrm{~K}$. An atomic pair distribution function approach evidences local symmetry breaking pre-existing far above the transition. The analysis shows the dimers evolve on heating into a short-range orbital degeneracy lifted (ODL)[1] state of dual orbital character, present up to at least $490 \mathrm{~K}$. The ODL state is correlated over the length scale spanning $\sim 6$ sites of the Ti zigzag chains. Our results imply that the ODL phenomenology extends to strongly correlated electron systems.

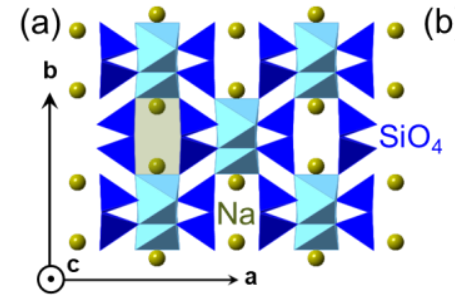

(c)

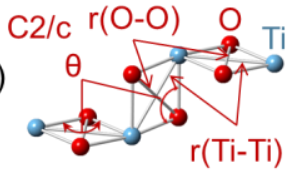

(b)

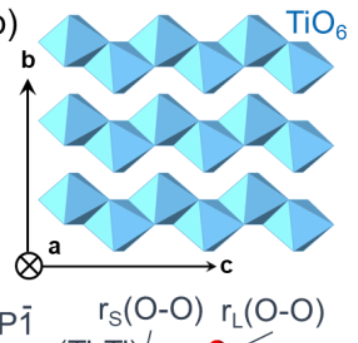

(d)

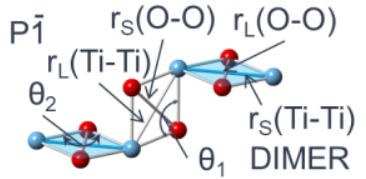

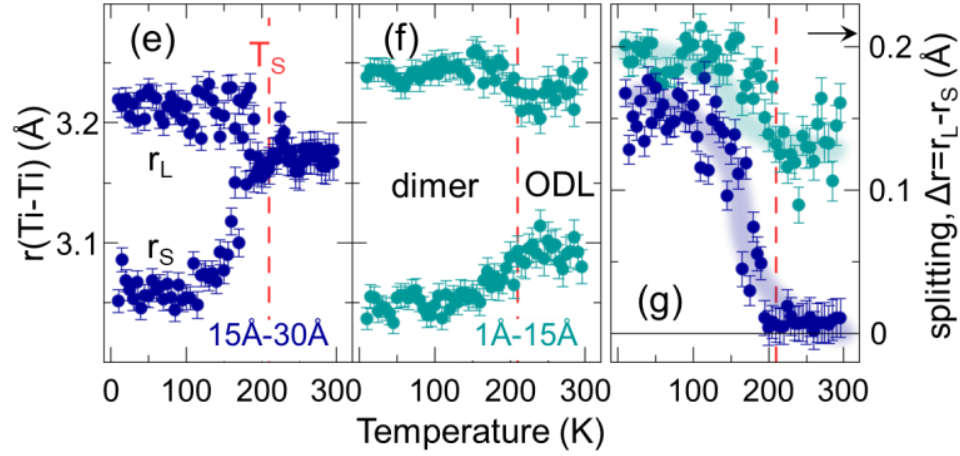

Figure 1. (a) $C 2 / c$ structure of $\mathrm{NaTiSi}_{2} \mathrm{O}_{6}$ (b) Quasi-1D zigzag $\mathrm{TiO}_{6}$ chains; (c) undistorted $\mathrm{TiO}_{2}$ plaquettes of the $C 2 / c$ phase, with uniform Ti-Ti and O-O distances; (d) distorted $\mathrm{TiO}_{2}$ plaquettes of the dimerized $\mathrm{P} \overline{\mathbf{1}}$ phase, with Ti-Ti and O-O distances split

$(\mathrm{S}=$ short, $\mathrm{L}=$ long). The nearest neighbour Ti-Ti distances from $\mathrm{P} \overline{\mathbf{1}}$-based model fits over (e) $15-30 \AA$ and (f) $1-15 \AA$ ranges. The assocaited $r(\mathrm{Ti}-\mathrm{Ti})$ splitting are shown in $(\mathrm{g})$.

[1] Bozin, E. S., Yin, W. G., Koch, R. J., Abeykoon, M., Hor, Y. S., Zheng, H., Lei, H. C., Petrovic, C., Mitchell, J. F. \&. Billinge, S. J. L (2019). Nat. Comm. 10, 3638.

Keywords: local structure; pair distribution function; total scattering

Work at Brookhaven National Laboratory was supported by U.S. Department of Energy, Office of Science, Office of Basic Energy Sciences (DOE-BES) under contract No. DE-SC0012704.

R.S. and H.Z. thank the support from the U.S. Department of Energy under award DE-SC-0020254. Neutron total scattering data were collected at the NOMAD beamline (BL-1B) at the Spallation Neutron Source, a U.S. Department of Energy Office of Science User Facility operated by the Oak Ridge National Laboratory.

$X$-ray PDF measurements were conducted on beamline 28-ID-1 of the National Synchrotron Light Source II, a U.S. Department of Energy (DOE) Office of Science User Facility operated for the DOE Office of Science by Brookhaven National Laboratory under Contract No. DE-SC0012704. 